\title{
A Kind of Seabed Seismic Data Acquisition Cell for Acoustic Measurement
}

\section{Guofang Dong 1}

National Engineering Laboratory for Vacuum Metallurgy,/ey Laboratory for Nonferrous Vacuum Metallurgy of Yunnan Province/hool of Metallurgy and Energy Engineering Kunming University of Science and Technology, Kunming 650093, China

School of Electrical and Information Technology, Yunnan Minzu University, Kunming 650031, China

E-mail: dongguofang1@163.com

\section{Bin Yang}

National Engineering Laboratory for Vacuum Metallurgy,/ey Laboratory for Nonferrous Vacuum Metallurgy of Yunnan Province/School of Metallurgy and Energy Engineering Kunming University of Science and Technology,

Kunming 650093, China

\section{Chuanxi Xing}

School of Electrical and Information Technology, Yunnan Minzu University

Kunming 650031, china

\section{Wenbo Shi}

School of Computer and Communication Engineering, Northeastern University at Qinhuangdao, Qinhuangdao, 066000,china

\begin{abstract}
This paper designs and realizes a low-power and high-resolution OBS sampling unit which can adopt 4-way synchronous sampling signals and store data in SD card. What's more, the system extends USB Host interface to connect with mobile hard disk in order to store data when there is a long time sampling. It realizes the two characters of low-power and dynamic range by carefully design its hardware structure and software digital signal processing algorithm. This sampling unit finally realizes dynamic range about $120 \mathrm{~dB}$ and when sampling ratio is less than $1 \mathrm{Ksps}$ and the power is less than $250 \mathrm{~mW}$ which ensures that battery can work long time undersea. Multiple trials verify the performance of sampling unit and compare its data with standard sampling device data. Experimental results demonstrate that the system utilizing over-sampling technology can improve dynamic range of signals. Besides, it verifies the reliability and practicability of the system by sampling of sound explosion signal further according to sea trials.
\end{abstract}

\section{CENet2017}

22-23 July 2017

Shanghai, China

${ }^{1}$ This study was supported by the Application Foundation Research Project of Yunnan Science and Technology Department (No. 2011FZ168), Yunnan Education Department Scientific Research Fund Project (2016ZZX128), the National Science foundation of China (No. 61461056, 61472074, 61662089). 


\section{Introduction}

With the application and popularization of vibration detecting technology, electricity technology and signal processing technology, people's demand for performance of vibration analyzer is becoming increasingly higher. It clearly manifests in vibration detecting field undersea, countries all over the world take lots of researches and experiments even invest rich human and material resources on the ocean bottom seismograph to detect vibration undersea.

The ocean bottom seismograph (OBS) is an earthquake observation system which is placed on the ocean floor directly. The observations are not only used to detect the natural earthquake, ocean artificial seismic section, whose measuring results are used to study dynamics features of oceanic crust, subduction zones, trench, trough, but also used on studying the natural earthquake seismic tomography and seismic activity and earthquake prediction. Currently, the U.S. and Japan have the leading position in this technology area [1-3].

Due to the particularity of the undersea earthquake observation, OBS data acquisition equipment must be has the characteristics of large dynamic-range, high-resolution, small-size, light-weight, low-power consumption, large-storage capacity and a high-degree of automation .At present, the majority of accurate collection equipments in the prevailing market are unable to meet the demands of low-power and high-resolution, and the price is exorbitant [4, 5].

In this paper, Combining with the characteristics of seabed seismic surveys and the development of the acquisition device, a new type of low-power, high-performance, highresolution seabed seismic acquisition cell is designed. The main contribution of this system include a 24-bit ADC which the dynamic range greater than $120 \mathrm{~dB}$; 4-way synchronous data acquisition channels which able to simultaneously the $\mathrm{X}, \mathrm{Y}, \mathrm{Z}$ three-way and all the way to low frequency sound pressure hydrophone device for data collection. What's more, its storage medium selects a $32 \mathrm{G}$ capacity SD card, and the external expansion of mobile hard disk. Furthermore, power consumption is less than $250 \mathrm{~mW}$ (when the sampling rate is less than $1 \mathrm{ksps})$.

\section{Whole Design}

The main function of this system includes 4-way synchronous data acquisition channels, which connect three-way OBS sensors and a sound pressure hydrophone device. Received sampling data stores in SD card or external hard disk; GPS interface is available; attitude sensor acquires attitude and azimuth of OBS undersea; consumption budget is less than $250 \mathrm{~mW}$ when normally used because of long time recording [6].

To make signal collection device has higher resolution and signal-to-noise ratio (SNR), suitable ADC needs to be chosen. According to sensitivity of OBS, sampling device needs the dynamic range greater than $120 \mathrm{~dB}$ and low-power. $\Sigma-\Delta \mathrm{ADC}$ is the candidate, due to a combination of factors. The $\Sigma-\Delta$ ADC is made up of $\Sigma-\Delta$ modulator and digital filter, it uses over-sampling technology to acquire higher signal-to-noise ratio. Over-sampling technology is that when sampling with high sampling rate, total energy of noise is unchanged, but distribution of noise has a great change. Quantizing noise will be pushed to high frequency part which can be reduced by digital filter. Based on Nyquist-Shannon sampling theorem, cut-off frequency of digital filter should be set half of sampling ratio which needs to be realized. According to oversampling theorem, sampling ratio rises every 2 times, sample-to-noise ratio will raise $3 \mathrm{~dB}$ [7]. 
The system is designed based on analysis of function needs. 24-bit $\Sigma-\Delta \mathrm{ADC}$ is chosen to satisfy with the demanding for dynamic range. What's more, it also needs 4-way synchronous data acquisition channels. Thinking about feature of low-power, ADS1274 of TI Company is chosen [2,3]. Power of every channel of ADS1274 is $7 \mathrm{~mW}$ when sampling ratio is $10 \mathrm{Ksps}$ and signal-to-noise ratio is $107 \mathrm{~dB}$. Moreover, rising 2 times of sample ratio can make signal-tonoise ratio rise $3 \mathrm{~dB}$. Therefore, 16 times sampling ratio can realize about $120 \mathrm{db}$ digital signal. For example, utilizing 16Ksps sampling ratio on digital-to-analog conversion can get $1 \mathrm{Ksps}$ digital signal whose signal-to-noise ratio is $120 \mathrm{db}$, through sample technology calculation. The highest sample ratio of ADS1274 can reach $128 \mathrm{~K}$ so that it can realize the highest $8 \mathrm{Ksps}$ digital signal whose signal-to-noise ratio is $120 \mathrm{db}$. The calculation of over-sampling part is finished by microprocessor control unit, so that a high-performance, high-speed and low-power microprocessor need to be chosen as system main control chip [8].

Combine with the characteristics above, this system chooses ARM CortexTM-M3 microprocessor as main control unit which controls digital-to-analog conversion chip sampling, it reads transformed data, and realizes digital filter and over-sampling calculation. It saves or transforms data in time, in addition, it connects external device (such as GPS) to make sure the whole system running normally [9].

Data storage part must be satisfied with demand of long time sampling. System configures a SD card, where data and system manifest files can be saved in and it can be directly visited by PC through FAT file system. This system chooses 32G SD card. Meanwhile, in order to satisfy with demand of more data collection, this system also extends a USB HOST full speed interface, and transplants FAT file system, so data can be stored in external mobile hard disk through the interface $[10,11]$.

\section{Hardware Design}

The structure schematic diagram of this system is shown in Fig.1. This system is made up of 4 differential input channels, microprocessor chip, storage unit, digital compass and power. Microprocessor not only connects to AD through SPI interface and generates CLK pulse signal for AD through inner timer but also connects to SD card through 4-way SDIO interface and extends a USB HOST full speed interface to connect with external mobile hard disk. Digital compass connects to main control chip through a group of TTL UART.

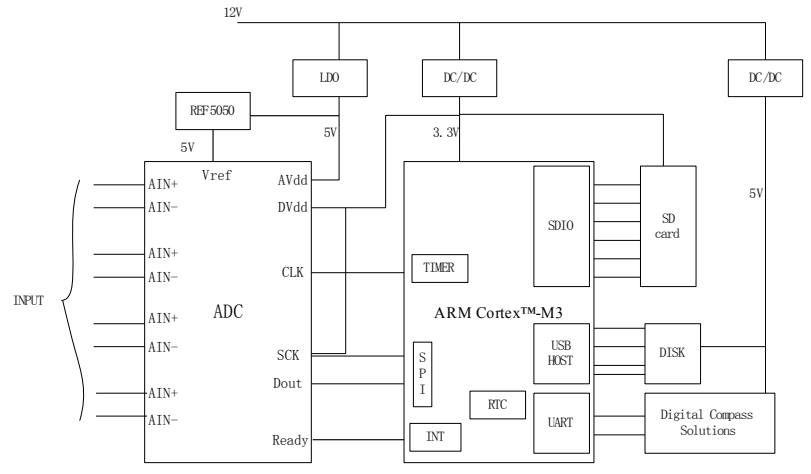

Figure 1: Structure of System

This system adopts $12 \mathrm{~V}$ lithium battery to product power and there are three level switch methods. Because demand of $5 \mathrm{~V}$ power of artificial part for current is lower and for noise is demanding, it can be got by extremely low noise LDO. $5 \mathrm{~V}$ and $3.3 \mathrm{~V}$ power of digital parts can 
be got by two groups of high-efficiency, high-performance switch buck DC-DC converter. All output powers are digital processed by RC net filter and decoupling capacitor.

Combine with analysis above, $\Sigma-\Delta$ ADC of this system adopts high-performance 24bit ADC ADS1274, which is the newest high-speed, 4-way synchronous data acquisition channels, 24bit ADC of TI Company's and its largest sampling ratio can reach $128 \mathrm{Ksp}$. What' more, it provides 8 bit parallel data interface and SPI interface. It can realize sampling function by controlling the logic pins of chip. When ADS1274 is running, modulator samples input signal through over-sampling ratio and according to over-sampling theorem, quantizing noise is pushed into high frequency part and then reduced $100 \mathrm{~dB}$ by inner digital filter. Over-sampling ratio is decided by operation mode of ADS1274, which are four modes: high-precision, highspeed, low-power and low-speed. These four modes can be switched by main control chip. Over-sampling ratio of modulator under high-precision, low-speed and low-power modes are 64 times and 128 times under high-speed mode. The largest sampling ratio, signal-to-noise ratio and noise under four modes are shown in Tab.1. In reality application, it can be adjusted based on reality needs.

The data output of ADS1274 has two modes: SPI and frame synchronization, and output forms are distribution output and stationary position output. In this system, microprocessor chip owns SPI interface so that it adopts SPI dynamic distribution output. The advantage of this output mode is that when using parts sampling channels, output data will be successively outputted dynamically and unused channels won't occupy bandwidth. ADS1274 needs a clock pulse as CLK, which is provided by frequency demultiplication of microprocessor inner timer. Changing frequency of this clock pulse can change sampling ratio. ADS1274 still needs a reference voltage which is very important, because performance of ADC basically is determined by stability of this reference voltage. This system adopts linear voltage regulator REF5050, which has advantages of low-noise, low-drifting and low-power-consumption. Circuit of output part is shown in Tab.1, aiming to further reduce hot noise of chip and improve precision and stability of reference voltage.

\begin{tabular}{|l|c|c|c|}
\hline Operating mode & maximum sampling rate & SNR & 8.5 \\
\hline High Precision & 128,000 sps & 106 & 8.5 \\
\hline high speed & 52,734 sps & 107 & 8 \\
\hline Low power consumption & 52,734 sps & 106 & 8.5 \\
\hline Low Speed & 10,574 sps & 107 & 8 \\
\hline
\end{tabular}

Table:Operating Mode Performance Summary

There are two ways to get the ADS1274's data output, SPI and frame synchronization scheme .And the output form is divided into dynamic allocation output and fixed position output .The system comes with microprocessor chips with SPI interface, so the dynamic allocation method used by SPI output. The benefits of using this output is that, when only part of the acquisition channel be used, the data will be dynamically output sequentially and output channels which is not used will no longer occupy the output bandwidth [4]. ADS1274 do need a clock pulse CLK, the clock pulse generated by microprocessor chip internal timer output frequency division, it also can change the sampling rate by changing the clock frequency. ADS1274 also requires a reference voltage which is very important, because the module conversion performance of ADC depends on the stability of the reference .Linear regulators REF5050 is adopted to implement in this system, REF5050 has the characteristics of low noise, low drift, and almost negligible loss of power. The circuit shown in Fig 2 designed in the output 
part in order to further thermal noise attenuation chips and improve the precision and stability of the reference level.

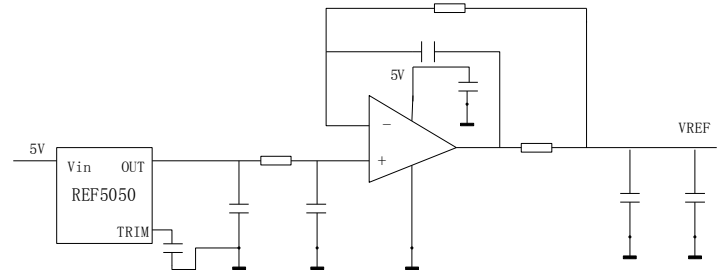

Figure 2: External Reference Driver

Peripheral of microprocessor includes SDIO interface that can connect SD card commodiously. Meanwhile, it employs extend USB HOST interface in order to connect mobile hard disk when needed. Both of two storage methods undergo the transplantation of FAT file system so that data can be directly identified and processed on PC.

To acquire attitude information of OBS undersea, digital compass is set in system which sends attitude information to microprocessor chip through UART interface of TTL. Microprocessor will store these data. In order to economize power consumption, digital compass is closed when OBS is stable undersea.

There is a RTC module in microprocessor chip, which used for mark date, system designs GPS clock configuration for it. To ensure precision of date, the system adopts GPS pulses per second (PPS) as time starting source. The first PPS triggers microprocessor which receives date information putted into register form asynchronous serial interface (ASI). At rising edge of fallowing second PPS, RTC register pluses 1 and finishes time service so there is no need to wait date information from ASI.

\section{Firmware Program Design}

Firmware of data sampling device is mainly consist of a main function and a high priority interrupt service which is used to realize reading $\mathrm{AD}$ data so that this part program needs precious calculation time and be coded efficiently.

There will be a falling edge at Ready pin before ADS1274 outputs data. This system connects this pin with interrupt channel so it will enter into interrupt service program before outputting data and then interrupt service program reads data from AD. The flow chart of interrupt service program is shown in Fig 3. Program adopts 8bit receiving mode per time and receives total 12 times because of four 24bit AD transformation channels. Receiving data needs about $11 \mathrm{uS}$ only if an $\mathrm{AD}$ output as $9 \mathrm{Mb} / \mathrm{s}$, interrupt response time is about $4 \mathrm{uS}$ so interrupt service function will take $15 \mathrm{uS}$ at most. For the rest of the time, program is used to realize algorithm of over-sampling technology. Both GPS and host communication belongs to system guiding part before starting sampling. 


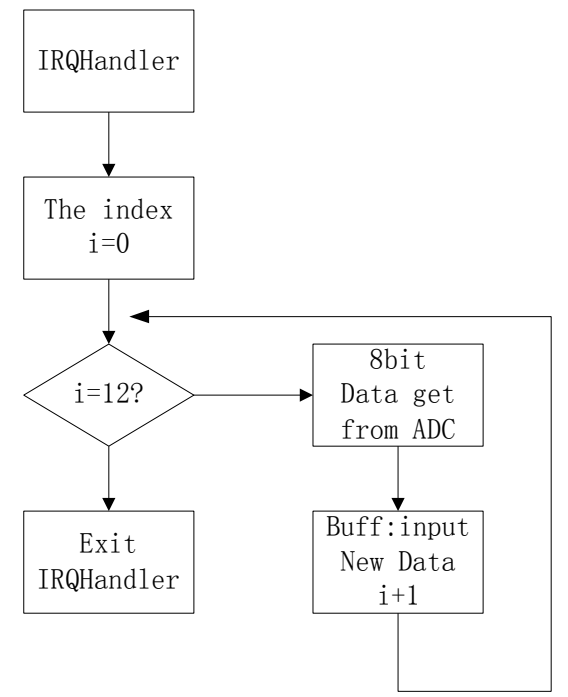

Figure 3:Flowchart of Interrupt Service Program

Sampling parameters should be initialized first after the system powered on. When system reaches sampling starting time, $\mathrm{AD}$ and interrupt service program will be started; data will be read. Received data is put into a buff array. When it reaches 8196 bytes, buffered data is calculated through sampling filter and then stored. SD card's writing is in the form of sector, each of which is 512 bytes and 8192 is 16 times as many as 512 . Moreover, a single file larger than 4GB is not allowed by FAT file system. It renames files and distributes store according to sampling times. Software flow chart of main program is shown in Fig. 4.

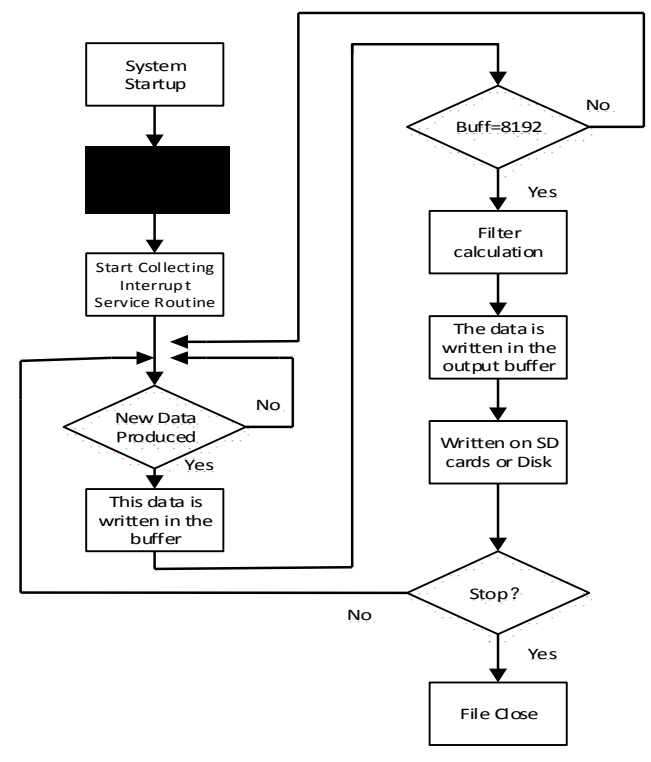

Figure 4: Software Flowchart of Main Program

Parameters setting of the system before starting can be set by external host through RS232 and also can be stored in SD card so as to call by system. When configured, system waits for external GPS synchronization pulse and synchronizes GPS time and RTC, and then GPS is closed after synchronization. Meanwhile, azimuth attitude information from digital compass is acquired and stored. After azimuth attitude information reaches a stable state, digital compass will be closed. 


\section{Experiment Result}

This system is tested to verify its technical specifications. Firstly, dynamic range of the system is verified. That is, signal period produced by signal generator is set $70 \mathrm{~Hz}$ and signal generator reduces signal amplitude to $10 \mathrm{uV}$ which is send to AD sampling channel of system through attenuator. Meanwhile, in order to compare the performance, signals are put into two systems to sample at the same time. One of the systems is OBS in this paper and another is standard sampling mode of ADS1274. Sampling rate of two systems is set as 500sps. Acquired data will be analyzed in time domain and frequency domain respectively.

Fig 5 shows the comparison of standard sampling mode and system in this paper in time domain. The picture below is oscillogram of this system, from which it is clearly identified its range and period. Power spectrum estimation of two groups' data is shown in Fig 6. The data SNR is higher than that of standard sampling mode $13 \mathrm{~dB}$. The picture exhibits that itself noise of this system is $-130 \mathrm{~dB}$. And it is unable to identify signal in time domain based on data from standard sampling mode.
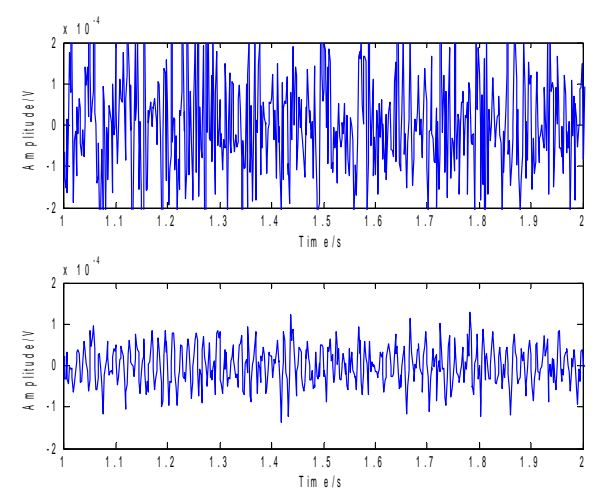

Figure 5: Sampled 70Hz Sinusoidal Signal
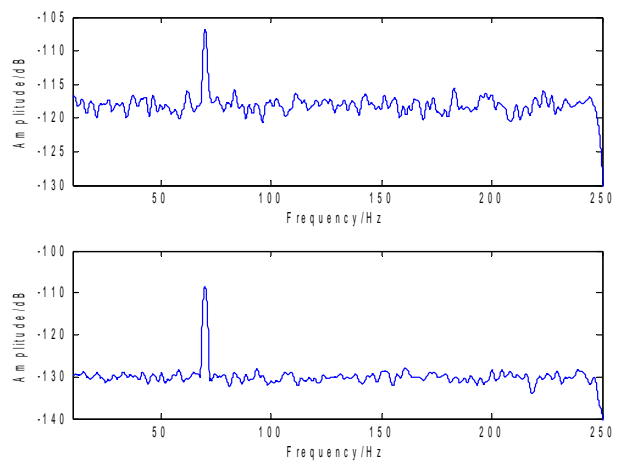

Figure 6:Sampled 70Hz Power Spectrum

Estimation

The system is tested in some area of Yellow Sea, China. 4-way sampling channels connect to a three-component geophone and a sound pressure sensor. Sampling unit and sensors are putted in a cylindrical volume which set under sea. Throw a sound bomb faraway from this area, sampling unit sensors receive signal of explosive sound.

In the sea trials, system sampling rate is set as $1 \mathrm{Ksps}$ and sound bomb is about $25 \mathrm{Km}$ away from sampling unit. One of explosive sound signal received is shown in Fig 7. Power spectrum is shown in Fig 8. In the picture, $\mathrm{X}, \mathrm{Y}, \mathrm{Z}$ is three directions of three-component geophone respectively. $\mathrm{P}$ is signal of sound pressure sensor.
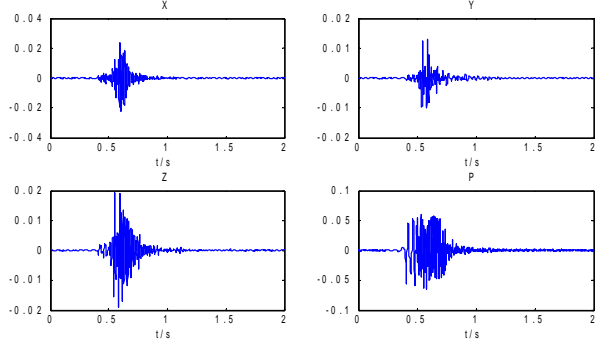

Figure 7: Time-domain Waveform of Explosive Sound Signal

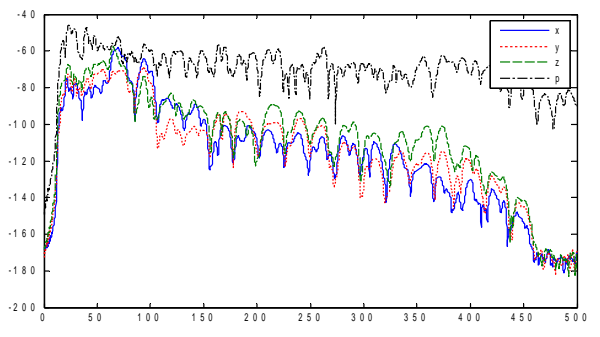

Figure 8:Power Spectrum Estimation of Explosive Sound Signal 
The acquisition unit adopts $12 \mathrm{~V}$ lithium battery to accommodate with power in the sea trials. In the process of long time running, average power has been calculated according to the battery discharge current. That is to say, it is about $237 \mathrm{~mW}$ which is satisfied with low-power requirement under low-speed mode (sampling ratio $<1 \mathrm{Ksps}$ ). While, under high-speed mode (sampling ratio $>1 \mathrm{Ksps}$ ), power is about $400 \mathrm{~mW}$.

\section{Conclusion}

This paper designs and realizes a low-power, high-performance and high-resolution synchronous data acquisition OBS unit with 4-channel whose dynamic range is larger than $120 \mathrm{~dB}$, storage medium is a $32 \mathrm{G}$ SD card and a external mobile hard disk to store data, total power is less than $250 \mathrm{~mW}$ when sampling ratio is less than $1 \mathrm{Ksps}$. Sea trials showed that, the system is reliable, hardware is uncomplicated and commodious. Therefore it can be wisely used in ocean vibration detection field.

\section{References}

[1] J. Kasahara, Geophysical observations at the ocean bottom, in: Proceedings of 2004 International Symposium on Underwater Technology, 20-23 April, 2004, 17-24.

[2] Texas Instruments Incorporated. ADS1294/ADS1298, Quad/Octal, Simultaneous Sampling, 24Bit Analog- to- Digital Package Datasheet [ODB/OL]. http://focus.ti.com/lit/ds/symlink/opa211.pdf,2010.

[3] Texas Instruments Incorporated. ADS1274/ADS1278, Quad/Octal, Simultaneous Sampling, 24Bit Analog- to- Digital Converters Datasheet [ODB/OL]. http://focus.ti.com/lit/ds/symlink/ads1274.pdf,2007.

[4] Gioacchino Fertitta, Antonio Di Stefano, Giuseppe Fiscelli, Giuseppe C. Giaconia, A low power and high resolution data logger for submarine seismic monitoring, Microprocessors and Microsystems, 2010,63-72.

[5] Cirrus Logic Inc. Reference Design: Multichannel Seismic Reference Design,CRD5376, 2007.

[6] STM32 RM0008 Reference Manual Datasheet[ODB /OL].http://www.st.com/stonline/products/literature/ds/14611.pdf. 2010.

[7] Franco.S.,Shudang Liu.Design with Operational Amplifiers And Analog Integrated Circuits[M]. Xian: XI'AN Jiaotong University Press, 2009. 277-289.

[8] IEEE Standard 1241-2000: IEEE Standard for Terminology and Test Methods for Analog-toDigital Converters, December 2000.

[9] Crystal Semiconductor Corporation Application Note: Delta Sigma A/D Conversion Technique Overview, January 1997.

[10] A.V. Oppenheim, R.W. Schafer, J.R. Buck, Discrete-Time Signal Processing, second ed., Prentice-Hall Inc., Upper Saddle River, NJ, 1999.

[11] National Marine Electronics Association: NMEA 0183 Standard v3.01, January, 2002. 